\title{
A new species and four newly recorded species of black flies (Diptera: Simuliidae) from Bhutan
}

\author{
Takaoka, H. ${ }^{{ }^{*}}$, Namgay, R. ${ }^{2}$, Somboon, P. ${ }^{3}$ \\ ${ }^{1}$ Tropical Infectious Diseases Research and Education Centre, Universiti Malaya, Kuala Lumpur, 50603, Malaysia \\ ${ }^{2}$ Vector-Borne Disease Control Programme, Ministry of Health, Gelphu, 31101, Bhutan \\ ${ }^{3}$ Department of Parasitology, Faculty of Medicine, Chiang Mai University, Chiang Mai, 50200, Thailand \\ *Corresponding author: takaoka@oita-u.ac.jp
}

\section{ARTICLE HISTORY}

Received: 20 September 2020 Revised: 27 November 2020

Accepted: 30 November 2020

Published: 25 March 2021

\begin{abstract}
Simulium (Simulium) thimphuense sp. nov. is described from a pupa and a mature larva in Bhutan. This new species is placed in the $S$. multistriatum species-group, and is characterized by the pupal gill with eight thread-like filaments divergent basally at an acute angle when viewed laterally and the cocoon slipper-shaped with several small openings anterolaterally. Four species of Simulium (Simulium) are newly recorded from Bhutan: S. barraudi Puri in the S. multistriatum species-group, S. nodosum Puri in the S. nobile species-group, S. chiangmaiense Takaoka \& Suzuki in the S. striatum species-group and S. himalayense Puri in the S. variegatum species-group. Our study increases the number of black fly species known from Bhutan from 18 to 23 .
\end{abstract}

Keywords: Simulium, blackfly, taxonomy, new species, Bhutan.

\section{INTRODUCTION}

The fauna of black flies in Bhutan is represented by 18 species, which are classified in four subgenera of the genus Simulium Latreille: two species in Gomphostilbia Enderlein, three species in Montisimulium Rubtsov, four species in Nevermannia Enderlein and nine species in Simulium Latreille (Lewis, 1974; Datta, 1991; Takaoka \& Somboon, 2008). Of the nine species in the subgenus Simulium, one is in the $S$. griseifrons species-group, one is in the $S$. indicum speciesgroup, two are in the $S$. multistriatum species-group, three are in the $S$. striatum species-group and two are in the $S$. tuberosum species-group.

We examined adults reared from pupae, pupal exuviae and larvae collected in 2007, 2016 and 2017 from Bhutan and found one new species and four newly recorded species. The new species is described based on a pupa and a mature larva and is placed in the $S$. multistriatum species-group. The four newly recorded species are each in the $S$. multistriatum species-group, S. nobile species-group, S. striatum speciesgroup, and $S$. variegatum species-group.

Methods of morphological observation, terms of features, descriptions, and illustrations, followed those of Takaoka (2003) and partially those of Adler et al. (2004).

The holotype and paratype of the new species are deposited in the Entomology Section of the Queen Sirikit Botanic Garden, Chiang Mai, Thailand.

Simulium thimphuense sp. nov.

Pupa $(\mathrm{n}=1)$. Body length $2.5 \mathrm{~mm}$. Head. Integument light ochreous, densely and uniformly covered with small tubercles (Figure 1A) except most of antennal sheath bare; frons with two unbranched slender short trichomes (Figure 1B) on each side; face with one unbranched slender long trichome (Figure 1C) on each side. Thorax. Integument light ochreous, densely and uniformly covered with small tubercles; thorax with three bifid or trifid long mediodorsal trichomes (Figure 1D), two unbranched anterolateral trichomes (anterior trichome medium-long and posterior one long) (Figure 1E), one unbranched medium-long mediolateral trichome (Figure 1F), and three unbranched ventrolateral trichomes (one mediumlong and two others long) (Figure 1G), on each side. Gill (Figure $1 \mathrm{H}, \mathrm{I}$ ) with eight slender thread-like short filaments arranged as $2+(2+2)+2$ from dorsal to ventral; middle two pairs sharing short stalk, and lying side by side; all pairs short-stalked; common basal stalk short; stalk of dorsal pair forming an angle of 40-80 degrees against stalk of ventral pair when viewed laterally; filaments decreasing in length and thickness from dorsal to ventral, with upper filament of dorsal pair longest (about $1.5 \mathrm{~mm}$ ) and lower filament of ventral pair shortest (about $0.8 \mathrm{~mm}$ ); relative basal thickness of eight filaments from dorsal to ventral 2.0:1.6:1.4:1.4:1.2:1.2:1.0:1.0; all filaments dark brown, tapered toward apex (though nearly parallel-sided from base for short distance), with distinct annular ridges and furrows, though annular ridges becoming less distinct apically, and densely covered with minute tubercles. Abdomen. Dorsally, all segments unpigmented and without microtubercles except segment 1 yellowish; segment 1 with one hair-like seta, though broken, on each side; segment 2 with one hair-like 
short seta, two slender minute setae, and three stout minute setae (Figure $1 \mathrm{~J}$ ), on each side; segments 3 and 4 each with four distinct hooked spines and one unbranched short seta on each side; segment 8 with distinct spine-combs and comb-like groups of microspines in transverse rows on each side; segments 5, 6, 7 and 9 lacking spine-combs; segment 9 without terminal hooks. Ventrally, segments 3-9 unpigmented, each (except segment 9) with comb-like groups of microspines; segment 4 with few unbranched slender minute setae on each side; segment 5 with a pair of bifid stout hooks submedially and few unbranched short setae on each side; segments 6 and 7 each with a pair of bifid inner and unbranched outer stout hooks somewhat separated from each other, and few unbranched short setae on each side. Cocoon (Figure $1 \mathrm{~K}$ ). Slipper-shaped, roughly woven, dark brown, with several small open spaces near anterior margin on each side, and not extended ventrolaterally; individual threads visible; $2.6 \mathrm{~mm}$ long by $1.2 \mathrm{~mm}$ wide.

Mature larva $(\mathrm{n}=1)$. Body length $4.5 \mathrm{~mm}$. Body dark gray. Head. Cephalic apotome medium brown with narrow portions along both lateral margins and along posterior margin dark brown, with indistinct head spots; lateral surface of head capsule medium brown except eyespot region light ochreous and narrow portion above eyespot region dark brown, and one small dark spot below eyespot region and one or two small dark spots near posterior margin somewhat distinct; ventral surface of head capsule medium to dark brown except narrow portion along posterior margin of hypostoma light ochreous. Labral fan with 48 primary rays. Antenna composed of three articles and apical sensillum, slightly longer than stem of labral fan; length ratio of three articles (from base to tip) 1.0:1.2:0.6. Mandible (Figure 2A) with mandibular serration composed of two teeth (one medium-sized, one small); main tooth at obtuse angle against mandible on apical side; comb-teeth gradually reduced in length from first to third; supernumerary serrations absent. Hypostoma (Figure 2B) with nine anterior teeth, of which median tooth slightly longer than corner teeth, followed by three intermediate teeth on each side; lateral margins serrate apically; six or seven hypostomal bristles divergent posteriorly from lateral border on each side. Postgenal cleft (Figure 2C) large, mitre-shaped; sheath of subesophageal ganglion moderately pigmented. Cervical sclerites on each side composed of light brown elliptical piece, not fused to occiput. Thorax and Abdomen. Histoblast of pharate pupal gill (Figure 2D) with eight thread-like slender filaments arranged in four pairs, of which two middle pairs overlapped. Thoracic and abdominal cuticle almost bare except last segment moderately covered with short colorless setae on each side of anal sclerite. Rectal scales distinct. Rectal organ of three lobes, each with 13 to 14 finger-like secondary lobules. Anal sclerite X-shaped, with short broad anterior arms about 0.6 times length of posterior arms and with forked apices. Last abdominal segment not bulged laterally and lacking ventral papillae. Posterior circlet with 86 rows of hooklets with up to 16 hooklets per row.

Type specimens. Holotype. Pupa, collected from grass in a fast running stream (partially shaded, elevation 2,391 m, $27^{\circ} 31^{\prime} 09^{\prime \prime} \mathrm{N}, 89^{\circ} 38^{\prime} 40^{\prime \prime} \mathrm{E}$ ), at Thimphu District, the capital of Bhutan, 17-IX-2016, by P. Somboon. Paratype. One mature larva, same data and date as for the holotype.

Etimology. The species thimphuense refers to the locality, Thimphu, where this new species was collected.

Remarks. Simulium thimphuense sp. nov. is placed in the S. multistriatum species-group of the subgenus Simulium, defined by Takaoka and Davies (1996), by having a com- bination of the pupal gill with eight filaments (Figure $1 \mathrm{H}, \mathrm{I}$ ) and pupal abdomen without terminal hooks.

This new species is distinguished from all 34 species of this species-group by the pupal gill with an acute angle between the stalks of the dorsal and ventral pairs of filaments when viewed laterally (Figure $1 \mathrm{H}, \mathrm{I}$ ) and cocoon slipper-shaped, with several small open spaces anterolaterally (Figure $1 \mathrm{~K}$ ). The known species of this species-group have the gill with a right or obtuse angle between the stalks of the dorsal and ventral pairs of filaments, and the cocoon slipper-shaped, with or without a relatively large open space, or shoe-shaped (Takaoka, 2017).

\section{Simulium barraudi Puri, 1932}

Simulium (Simulium) barraudi Puri, 1932b: 1130-1132 (Female, male and pupa); Takaoka et al., 2020 (Male and pupa).

Simulium sp. B Lewis, 1964: 293-294 (Female).

Specimen examined. One male reared from a pupa, collected from a moderately flowing stream (width $2 \mathrm{~m}$, $22.0^{\circ} \mathrm{C}$, exposed to the sun, elevation $2,570 \mathrm{~m}, 27^{\circ} 32^{\prime} 20^{\prime \prime} \mathrm{N}$, $\left.90^{\circ} 48^{\prime} 26^{\prime \prime} \mathrm{E}\right)$, Dungmithang, Bumthang District, Bhutan, 5-IX2007, by P. Somboon.

Distribution. Bhutan (New record), India, Myanmar, Nepal, Pakistan, Tibet.

Remarks. This species was originally described from India by Puri (1932b). It belongs to the S. multistriatum speciesgroup (Adler, 2020). The male and pupa of this species were redescribed by Takaoka et al. (2020).

The male of this species is characterized by the uppereye (large) facets in 19 vertical columns and 19 horizontal rows, scutum with fine yellow hairs, mid-femur entirely whitish yellow, ventral plate lacking ridges or teeth on the posterior surface and style broadest near its apex when viewed ventrolaterally.

The male examined is in good agreement with that in the redescription by Takaoka et al. (2020).

This species is recorded for the first time from Bhutan.

\section{Simulium nodosum Puri, 1933}

Simulium (Simulium) nodosum Puri, 1933: 813-816 (Female, pupa and larva); Lewis, 1974: 34-37 (Female, male, pupa and larva).

Specimens examined. Two males reared from pupae, and 11 pupae collected from grasses in a small slow running stream (exposed to the sun, elevation $220 \mathrm{~m}, 26^{\circ} 51^{\prime} 09^{\prime \prime} \mathrm{N}$, $90^{\circ} 31^{\prime} 59^{\prime \prime}$ E), at Umling, Sarpang District, Bhutan, 31-X-2017, by P. Somboon.

Distribution. Bhutan (New record), China (Fujian, Hainan, Hong Kong, Yunnan), India, Myanmar, Taiwan, Thailand, Tibet, and Vietnam.

Remarks. This species was originally described from India by Puri (1933). It belongs to the S. nobile species-group (Adler, 2020).

This species is characterized by the pupal gill with three inflated tubal filaments. The males and pupae examined agree with those in the original description.

This species is recorded for the first time from Bhutan.

Simulium chiangmaiense Takaoka \& Suzuki, 1984

Simulium (Simulium) chiangmaiense Takaoka \& Suzuki, 1984: 38-39 (Female, male, pupa); Takaoka et al., 2018: 285-288 (Pupa).

Specimen examined. One pupa collected from the same stream, from where $S$. nodosum was collected, at Umling, Sarpang District, Bhutan, 31-X-2017, by P. Somboon.

Distribution. Bhutan (New record), China (Yunnan), Myanmar, Thailand, and Vietnam. 


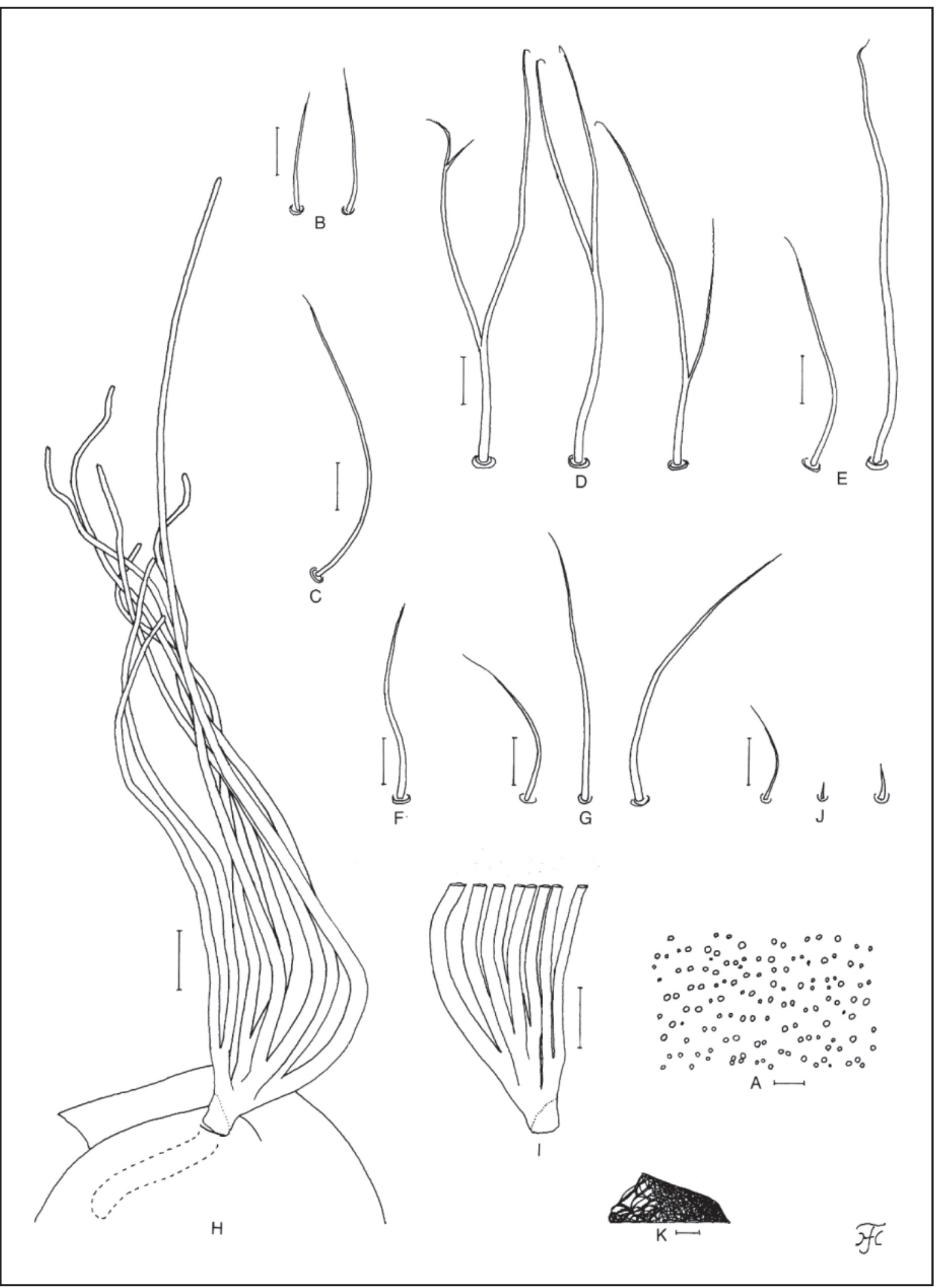

Figure 1. Pupa of Simulium thimphuense sp. nov. (A) Tubercles on frons. (B) Trichomes on frons. (C) Trichome on face. (D)-(G) Thoracic trichomes (D, mediodorsal; $E$, anterolateral; $F$, mediolateral; $G$, ventrolateral). (H) Anterior part of thorax and gill filaments (left side; lateral view). (I) Basal portion of gill filaments (right side; lateral view). (J) Hair-like seta, slender minute seta and stout minute seta on dorsum of abdominal segment 2. (K) Cocoon (lateral view). Scale bars. $0.5 \mathrm{~mm}$ for $\mathrm{K} ; 0.1 \mathrm{~mm}$ for $\mathrm{H}$ and I; $0.02 \mathrm{~mm}$ for B-G and J; $0.01 \mathrm{~mm}$ for A. 

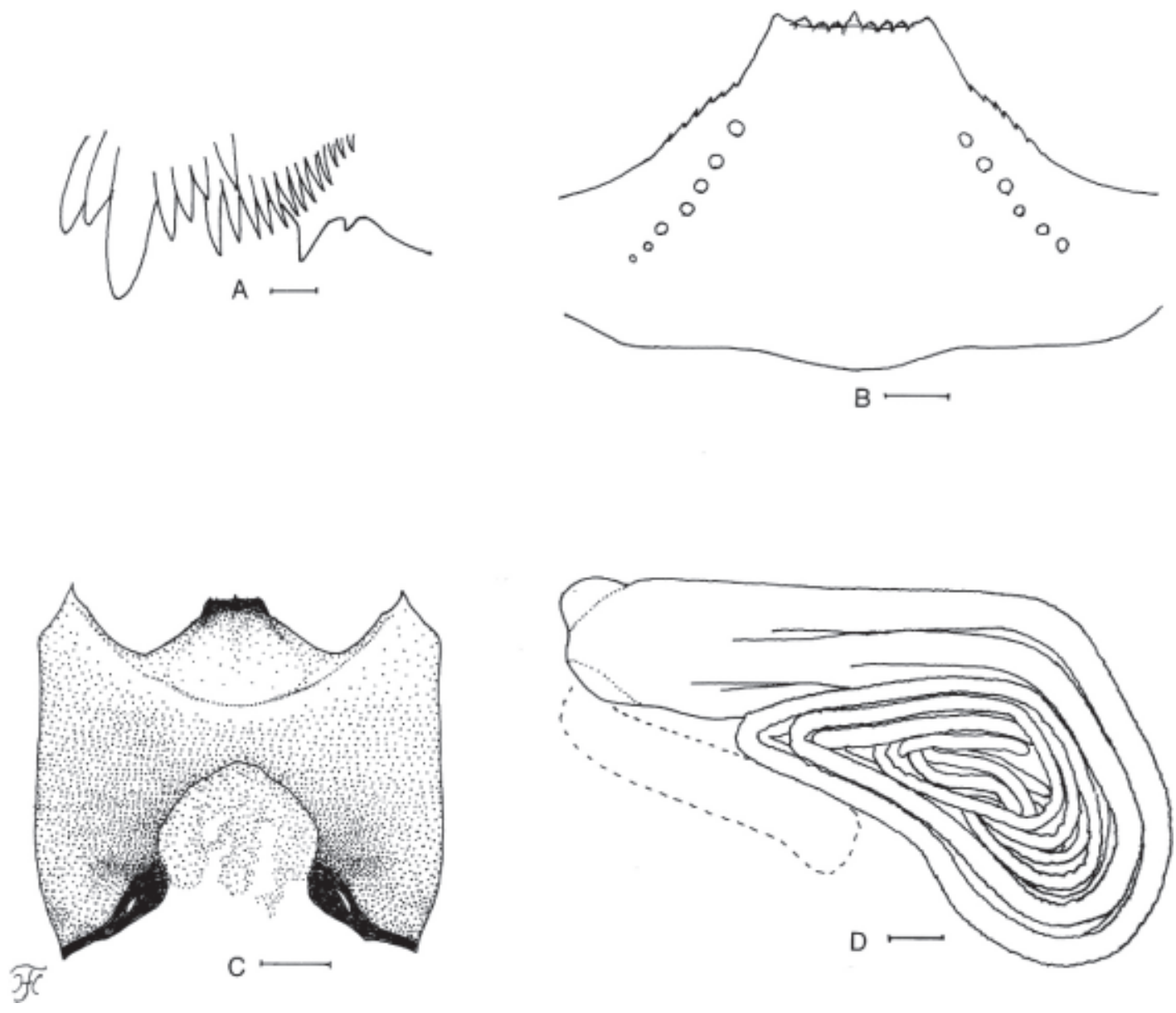

Figure 2. Mature larva of Simulium thimphuense sp. nov. (A) Mandible. (B) Hypostoma. (C) Head capsule (ventral view). (D) Histoblast of pupal gill filaments. Scale bars. $0.1 \mathrm{~mm}$ for $C ; 0.04 \mathrm{~mm}$ for $D ; 0.03 \mathrm{~mm}$ for $B ; 0.01 \mathrm{~mm}$ for $A$.

Remarks. This species was originally described from Thailand by Takaoka and Suzuki (1984). It belongs to the $S$. striatum species-group (Adler, 2020).

The pupa of this species is characterized by the head and thoracic integument densely covered with relatively large tubercles having secondary projections, and gill with eight filaments, of which six are relatively thicker than the remaining two filaments.

The pupa examined agrees with that in the original description. The pupa of $S$. kapuri in the $S$. striatum speciesgroup from Assam, India, has the gill with eight filaments but differs from that of $S$. chiangmaiense by lacking terminal hooks (Datta, 1975).

This species is recorded for the first time from Bhutan.

\section{Simulium himalayense Puri, 1932}

Simulium (Simulium) himalayense Puri, 1932a: 885-890 (Female, male and pupa); Datta, 1974: 21 (Larva).

Specimens examined. One female reared from a pupa, collected from grass in a fast running stream (elevation 2,307 $\left.\mathrm{m}, 27^{\circ} 23^{\prime} 07^{\prime \prime} \mathrm{N}, 8^{\circ} 25^{\prime} 02^{\prime \prime} \mathrm{E}\right)$, above Bonde, at Paro District, Bhutan, 25-X-2017, by P. Somboon; one female reared from a pupa, collected from grass in a small irrigation ditch in rice

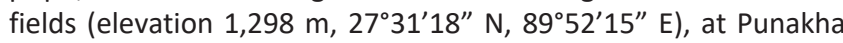
District, Bhutan, 24-X-2017, by P. Somboon; two pupae collected from grasses in a narrow concrete ditch (elevation 2,894 m, $27^{\circ} 29^{\prime} 18^{\prime \prime} \mathrm{N}, 89^{\circ} 43^{\prime} 26^{\prime \prime}$ E) at Honsa checkpoint, Thimphu District, Bhutan, 18-IX-2016, by P. Somboon.
Distribution. Bhutan (New record), India, Myanmar, Nepal, Pakistan, Tibet.

Remarks. This species was originally described from India by Puri (1932a). It belongs to the $S$. variegatum speciesgroup (Adler, 2020).

This species is characterized by yellowish female femora, integument of the pupal head and thorax moderately covered with tubercles, and pupal gill with six slender filaments divergent basally at an obtuse angle when viewed laterally.

The females and pupae examined agree with those in the original description.

This species is recorded for the first time from Bhutan.

\section{Conflict of Interest:}

The authors declare that they have no conflict of interest.

\section{ACKNOWLEDGEMENTS}

We are grateful to Dr. Peter H. Adler (Professor Emeritus, Clemson University, Clemson, SC, USA) for reading the current manuscript and providing valuable comments. Thanks are due to Dr. M. Fukuda, Oita University, for her kind help in various ways. This research was funded by Faculty of Medicine (Grant No. PAR-2563-07268) and Office of Research Administration, Chiang Mai University. This study was funded by the Higher Institution Center of Excellence (HICoE) program (MO002-2019). 


\section{REFERENCES}

Adler, P.H. (2020). World Blackflies (Diptera: Simuliidae): A Comprehensive Revision of the Taxonomic and Geographical Inventory [2020]. pp. 142. https://biomia.sites. clemson.edu/pdfs/blackflyinventory.pdf

Adler, P.H., Currie, D.C. \& Wood, D.M. (2004). The Black Flies (Simuliidae) of North America. xv+941 pp., Cornell University Press, Ithaca, New York.

Datta, M. (1991). A preliminary report on the black flies (Diptera: Simuliidae) from Bhutan. Journal of Bengal Natural History Society, New Series 10: 29-37.

Datta, M. (1974). New species of black flies (Diptera: Simuliidae) from the Darjeeling area, India. Oriental Insects 8: 457-468.

Datta, M. (1975). Simuliidae (Diptera) from Assam foot-hills, India. Japanese Journal of Sanitary Zoology 26: 31-40.

Lewis, D.J. (1964). Diptera from Nepal. Simuliidae. Bulliten of British Museum (Natural History) 15: 291-294.

Lewis, D.J. (1974). Man-biting Simuliidae (Diptera) of Northern India. Israel Journal of Entomology 9: 23-53.

Puri, I.M. (1932a). Studies on Indian Simuliidae. Part I. Simulium himalayense sp. n.; Simulium gurneyae Senior-White; and Simulium nilgiricum sp. $\mathrm{n}$. Indian Journal of Medical Research 19: 883-898.

Puri, I.M. (1932b). Studies on Indian Simuliidae. Part III. Descriptions of males, females, and pupae of Simulium griseifrons Brunetti (1911), and of four new species with striped thorax. Indian Journal of Medical Research 19: 11251143.
Puri, I.M. (1933). Studies on Indian Simuliidae. Part VII. Descriptions of larva, pupa and female of Simulium nodosum sp. nov. with an appendix dealing with $S$. novolineatum nov. nom. ( $=S$. lineatum Puri). Indian Journal of Medical Research 20: 813-817.

Takaoka, H. (2003). The Black Flies (Diptera: Simuliidae) of Sulawesi, Maluku and Irian Jaya. xxii + 581 pp., Kyushu University Press, Fukuoka.

Takaoka, H. (2017). Morphotaxonomic revision of speciesgroups of Simulium (Simulium) (Diptera: Simuliidae) in the Oriental Region. Zootaxa 4353: 425-446. https://doi.org/ 10.11646/zootaxa.4353.3.2

Takaoka, H. \& Davies, D.M. (1996). The Black Flies (Diptera: Simuliidae) of Java, Indonesia. viii +81 pp., Bishop Museum Bulletin in Entomology 6, Bishop Museum Press, Honolulu.

Takaoka, H., Shrestha, S. \& Dangi, N. (2020). Three new species and one newly recorded species of the Simulium (Simulium) multistriatum species-group (Diptera: Simuliidae) from Nepal. Medical Entomology and Zoology 71: 289-306. https://doi.org/10.7601/mez.71.289

Takaoka, H. \& Somboon, P. (2008). Eleven new species and one new record of black flies (Diptera: Simuliidae) from Bhutan. Medical Entomology and Zoology 59: 213-262. https://doi.org/10.7601/mez.59.213

Takaoka, H., Srisuka, W., Maleewong, W. \& Saeung, A. (2018). New record of 13 species of black flies (Diptera: Simuliidae) from Myanmar. Acta Tropica 178: 281-289. https://doi.org/10.1016/j.actatropica.2017.12.009

Takaoka, H. \& Suzuki, H. (1984). The blackflies (Diptera: Simuliidae) from Thailand. Japanese Journal of Sanitary Zoology 35: 7-45. https://doi.org/10.7601/mez.35.7 